\title{
Correlation between Visual Field Changes and Peripapillary Vessel Density in Primary Open Angle Glaucoma
}

\author{
Ahmed Fouad Ibrahim ${ }^{1}$, Zeinb Sayed Hassan ${ }^{2}$, Nesma Sayed Mohammed ${ }^{* 2}$ \\ ${ }^{1}$ Department of Ophthalmology, Magrabi Eye Hospitals, and ${ }^{2}$ Department of Ophthalmology, Faculty of \\ Medicine (For Girls), Al-Azhar University, Cairo, Egypt \\ *Corresponding author: Nesma Sayed Mohammed, Mobile: (+20)1014852522, E-mail: nesma7@live.com
}

\begin{abstract}
Background: Glaucoma is a progressive optic neuropathy, which is characterized by degeneration of the retinal ganglion cells. The role of optic nerve head $(\mathrm{ONH})$ microvascular network and vascular dysfunction has been investigated in the pathogenesis of glaucoma.

Objective: The aim of the current work was to assess the correlation between visual field changes in primary open angle glaucoma (POAG), and the density of the radial vessels around the optic disc.

Patients and Methods: One hundred eyes of fifty patients were enrolled in this prospective cross-sectional study. Patients were recruited from the Department of Ophthalmology, Al-Zahraa University Hospital from December 2018 to March 2020. They were divided into two groups, control group with normal functional and structural data, and glaucomatous group with POAG.

Results: There were significant differences in all measurements among both groups, including visual field indices, optical coherence tomography (OCT) and optical coherence tomography angiography (OCTA) parameters. The changes of peripapillary vessel density (PpVD) had strongly positive correlations with the mean deviation (MD), ganglion cell complex (GCC) thickness and retinal nerve fiber layer (RNFL) thickness, while had negative correlation with pattern standard deviation (PSD) and cup disc (C/D) ratio.

Conclusion: Decreased PpVD was detected in the glaucomatous group. There were statistically significant correlations between visual field changes (changes of both MD and PSD) and PpVD where there were strongly positive correlations with the (MD), and negative correlation with PSD. This can add to the diagnosis of POAG. Also, it provided more information about the microvascular changes in the $\mathrm{ONH}$ and peripapillary retina in cases of POAG.
\end{abstract}

Keywords: Optical coherence tomography angiography, Ganglion cell complex, Retinal Nerve fiber layer.

\section{INTRODUCTION}

Glaucoma is a group of progressive optic neuropathies characterized by a degeneration of retinal ganglion cells and retinal nerve fiber layers that result in specific changes in the optical nerve head $(\mathrm{ONH})^{(\mathbf{1})}$. It is well documented that early diagnosis, early treatment and monitoring of the disease progression are the key determinants to reduce the risk of irreversible vision loss ${ }^{(2)}$. Although the pathogenesis of glaucoma is not fully understood, it is well known that structural damage occurs prior to functional damage ${ }^{(3)}$.

It may be not appropriate to diagnose the earliest stages of glaucoma with visual field (VF) testing because a significant amount of ganglion cell (GC) loss is predicted to occur before the functional deficits start to appear ${ }^{(4)}$. Peripapillary retinal nerve fiber layer (RNFL) thinning which is better detected using optical coherence tomography (OCT) system, ganglion cell complex GCC thickness reduction and neuroretinal rim narrowing are other available good parameters for diagnosing the early changes and monitoring the progression of glaucoma, while detection of VF defects is indispensable for monitoring the functional decline. Some studies have suggested that reduced ocular blood flow is a primarily independent metric of visual function outside of other structural parameters, supporting a vascular role in the development of glaucoma ${ }^{(5,6)}$.

Optical coherence tomography angiography (OCTA) has several advantages over the other previous devices since it is a non-invasive, reliable, depthresolved, and user-friendly technique. Since our knowledge has been started to expand about the role of ocular blood flow in glaucoma, OCTA is expected to provide us with qualitative and quantitative information of the microvasculature in various retinal regions including the optic nerve and peripapillary retina ${ }^{(7)}$. Also, we can easily get insight of the relationship between retinal blood flow and visual functional damage ${ }^{(8)}$.

The aim of this study was to correlate between peripapillary vessel density and glaucomatous visual field changes in primary open angle glaucoma (POAG), and to evaluate the diagnostic abilities of measuring the peripapillary vessel density in the diagnosis of POAG.

\section{PATIENTS AND METHOD}

This observational prospective, cross sectional study included a total of one hundred eyes of fifty persons attending at the Department of Ophthalmology, Al-Zahraa University Hospital. This 
study was conducted between December 2018 to March 2020.

\section{Ethical Consideration:}

An informed written consent was obtained from each participant after receiving a full explanation about the study. Approval was obtained from the Ethics Board of Al-Azhar University committee. All procedures were conducted in accordance with the Helsinki Declaration guidelines.

The included subjects were divided into two equal groups, 25 each; Group 1 (control) included those subjects without family history of POAG, intraocular pressure (IOP) less than $21 \mathrm{~mm} \mathrm{Hg}$, open angles on gonioscopy, normal anterior and posterior segment on slit-lamp examination, normal optic discs, Group 2 (POAG group) included those that had IOP more than $21 \mathrm{mmHg}$, open angles on gonioscopy, glaucomatous optic disc changes, and glaucomatous visual field defect.

Exclusion criteria were the presence of retinal or macular pathology, non-glaucomatous optic neuropathy, and any systemic or neurological conditions that could produce VF defects. Uncooperative patients for both $\mathrm{VF}$ test and OCT/OCTA scanning were also excluded. All participants underwent a comprehensive ocular examination, which included corrected distance visual acuity measurement, slit-lamp biomicroscopy, Goldmann applanation tonometry, gonioscopy, fundus examination, VF testing, and OCT / OCTA scanning.

Humphrey visual field (VF) test: VF test was performed with the Swedish Interactive Threshold Algorithm (SITA) Standard strategy program 24-2 of the Humphrey Field Analyzer II (Carl Zeiss Meditec, Jena, Germany). The reliable field was considered if fixation losses that were not more than $20 \%$, false positives, and false negatives not more than which were not more $33 \%$. A normal VF was defined as a pattern standard deviation (PSD) within the 95\% confidence limits and a Glaucoma VF test within normal limits. Glaucomatous VF defects were considered if there were a group of three non-edged points with $\mathrm{P}<0.05$ on the pattern deviation map, including 1point with $\mathrm{P}<$ 0.01 , a PSD of $\mathrm{P}<0.05$, or if the VF analysis were outside the normal limits.

Spectral Domain OCT Imaging: Optic nerve head and macular imaging were also performed using the spectral domain -optical coherence tomography system on the same day as OCTA imaging and by the same operator. Avanti OCT uses a light source with center wavelength of $840 \mathrm{~nm}$, and it has an A-scan rate of 70$\mathrm{kHz}$. The ONH scanning protocol was used for measuring the RNFL thickness and the macular ganglion cell complex (GCC) scanning protocol was utilized to measure the GCC thickness.
Specifically, the ONH map protocol calculates circumpapillary RNFL thickness in a 10 pixel-wide band along a circle of $3.45 \mathrm{~mm}$ in diameter centered on the ONH based on 360-degree global area and eight 45degree sectors. Global average measurements were used for the analysis in the present study.

The GCC scanning protocol consists of a $7 \times 7 \mathrm{~mm}^{2}$ raster scan consisting of 1 horizontal B scan of $933 \mathrm{~A}$ scans, 15 vertical B scans of 933 A-scans per B-scan. This protocol measures retinal thickness from the ILM to the IPL posterior boundary obtained from the raster scans and GCC thickness measurements consist of the ganglion cell layer, IPL and RNFL. The mean GCC thickness measurements were used for the analysis. Only good-quality ONH and GCC images, defined by scans with $\mathrm{SSI} \geq 37$, and without segmentation failure or artifacts such as missing or blank areas were included for the analysis.

OCT Angiography Image Acquisition and Processing: All subjects underwent OCTA imaging with a commercially available OCTA system, the Angiovue that is incorporated in the Avanti OCT system (Optovue RTVue XR Avanti, Inc., Fremont, CA, USA). The Angiovue imaging system provides a non-invasive method of characterizing the vascular structures of the retina at the capillary level. It uses the Split-Spectrum Amplitude-Decorrelation Angiography (SSADA) algorithm to capture the dynamic motion of moving particles such as red blood cells and provides a high-resolution 3D angiogram of perfused retinal vasculature. The Angiovue characterizes vascular information at various user-defined retinal layers qualitatively as a vessel density map and color-coded vessel area density. Quantitatively it provides vessel density (\%) measurements calculated as the percentage of measured area occupied by flowing blood vessels being defined as pixels having SSADA algorithmbased decorrelation values above the threshold level. For the present study, peripapillary vessel density was derived from the images acquired with a $4.5 \times 4.5 \mathrm{~mm}$ field of view centered on the optic disc. Peripapillary vessel density PpVD measurements were calculated within the RNFL in a slab from the internal limiting membrane (ILM) to the RNFL posterior boundary.

Poor quality scans were excluded from the analysis if one of the following criteria were met: (1) Signal strength index (SSI) of lower than 48, (2) Poor clarity images, (3) Local weak signal caused by artifacts such as floaters, (4) Residual motion artifacts visible as irregular vessel patterns or disc boundary on the enface angiogram, and (5) RNFL segmentation failure.

\section{Statistical analysis}

Analysis of data was performed using software MedCalcv. 19. Description of variables was presented: Description of quantitative variables was in the form of mean, standard deviation (SD), minimum and 
maximum. Description of qualitative variables was in the form of numbers (No.) and percent's (\%). Data were explored for normality using KolmogorovSmirnov test of normality. Binary correlation was carried out by Pearson correlation test.

Results were expressed in the form of correlation coefficient $(\mathrm{R})$ and $\mathrm{P}$-values. $\mathrm{P}$ values equals or less than 0.05 was considered significant.

\section{RESULTS}

Demographic characteristics of glaucoma and control subjects:

Group1 (control) consisted of 21 males and 4 females. Group 2 (POAG) consisted of 14 males and 11 females. The mean age was 53.50 \pm 9.37 ranged from 40-81 years. Compared to the control group, MD was significantly lower with higher IOP and PSD in glaucoma group (Table 1).

Table (1): General ocular characteristics of the study groups.

\begin{tabular}{|l|c|c|c|c|c|}
\hline \multirow{2}{*}{$\begin{array}{l}\text { Groups } \\
\text { Ocular } \\
\text { measurements }\end{array}$} & \multicolumn{2}{|c|}{ POAG } & \multicolumn{2}{c|}{ Control } & \multirow{2}{*}{ Pean } \\
\cline { 2 - 5 } & SD & Mean & SD & \\
\hline BCVA & 0.86 & 0.21 & 0.93 & 0.09 & $0.046^{*}$ \\
\hline C/D ratio & 0.63 & 0.16 & 0.28 & 0.11 & $<0.001^{*}$ \\
\hline IOP & 19.02 & 2.60 & 12.88 & 1.94 & $<0.001^{*}$ \\
\hline
\end{tabular}

\section{Visual field, OCT, OCTA changes:}

There were significant differences in all measurements among the groups, including visual field indices (MD and PSD), OCT parameters (average RNFL thickness and GCC thickness), and PpVD with ( $\mathrm{p}<0.01)$. The average PpVD was $41.18 \pm 260$ in the POAG group, while the average PpVD was 56.83 \pm 3.22 in the control group (Table 2).

Table (2): Comparison between visual field, OCT\& OCTA variables between case and control by ANOVA test.

\begin{tabular}{|l|c|c|c|c|c|}
\multirow{2}{*}{ Groups } & \multicolumn{2}{c|}{$\begin{array}{c}\text { Case } \\
\text { (POAG) }\end{array}$} & \multicolumn{2}{c|}{ Control } & \multirow{2}{*}{ P-value } \\
\cline { 2 - 5 } Variables & Mean & SD & Mean & SD & \\
\hline MD & -6.04 & 4.53 & -0.89 & 0.50 & $<0.001^{*}$ \\
\hline PSD & 5.05 & 1.92 & 1.33 & 0.43 & $<0.001^{*}$ \\
\hline RNFL & 86.07 & 12.60 & 101.52 & 3.87 & $<0.001^{*}$ \\
\hline GCC & 87.27 & 11.17 & 94.84 & 2.38 & $<0.001^{*}$ \\
\hline PpVD & 41.18 & 2.60 & 56.83 & 3.22 & $<0.001^{*}$ \\
\hline
\end{tabular}

Correlation of PpVD with structural and functional data:

PpVD showed a significant positive correlation with MD, while negatively correlated with PSD and average
$\mathrm{C} / \mathrm{D}$ ratio $(\mathrm{R}+\mathrm{ve}, \mathrm{P}<0.05)$. $\mathrm{PpVD}$ was also positively correlated with GCC and RNFL thickness $(p<0.01)$ (Table 3).

Table (3): Pearson correlation between visual field \&OCT parameters and PpVD.

\begin{tabular}{|c|c|c|c|c|c|c|c|c|}
\hline \multirow[b]{2}{*}{ Correlations } & \multicolumn{2}{|c|}{ MD } & \multicolumn{2}{|r|}{ PSD } & \multicolumn{2}{|c|}{ RNFL } & \multicolumn{2}{|c|}{ GCC } \\
\hline & $\mathbf{R}$ & \begin{tabular}{c|} 
P- \\
value
\end{tabular} & $\mathbf{R}$ & P-value & $\mathbf{R}$ & $\begin{array}{c}\text { P- } \\
\text { value }\end{array}$ & $\mathbf{R}$ & $\begin{array}{c}\text { P- } \\
\text {.value }\end{array}$ \\
\hline PpVD & 0.516 & $\begin{array}{c}0.0003 \\
*\end{array}$ & $\begin{array}{c}- \\
0.93\end{array}$ & $\begin{array}{c}< \\
0.0001 *\end{array}$ & 0.245 & 0.1045 & 0.348 & 0.0191 \\
\hline
\end{tabular}

\section{DISCUSSION}

Prior studies have shown that glaucomatous damage can be detected by OCT as reduced RNFL thickness in the peripapillary region or thinning of the ganglion cell complex within the macula area $(\mathbf{9}, \mathbf{1 0})$. With the development of OCTA, more qualitative and quantitative information of various retinal regions could be obtained.

In the present study, we have found that both the functional parameters (MD and PSD) and structural parameters (mean RNFL and GCC) thickness were decreased in POAG group in comparison to the control group with a statistically significant difference between both the study groups. This was agreed with the standard diagnosis of glaucoma e.g, where reduced structural and functional parameters were confirmed ${ }^{(11}$, 12).

In our study, we also found that, POAG group showed a reduction in the PpVD in comparison to the control group. This agreed with Li et al. ${ }^{(13)}$ who concluded in their work that the more severe the glaucoma was, the lower the vessel density at the peripapillary region.

We also found that the reduced PpVD were significantly correspond with the severity of VF damage, which was in agreement with many other studies $(14,15,16)$.

Additionally, we found that there were statistically significant positive correlations between PpVD with both MD and rim area, while there were statistically significant negatively correlations between PpVD with both PSD and average C/D ratio, which meant, the more severe the glaucoma was, the lower PpVD was. Almbsut et al. ${ }^{(17)}$, in high agreement of our study results, found that the changes of PpVD may be better facilitated for the observation and monitoring of glaucoma progression.

Our study showed that PpVD were positively correlated with GCC and RNFL thickness. This was consistent with Yarmohammadi et al. ${ }^{(18)}$, who specifically compared the relation between PpVD with visual field indices and both RNFL \&GCC thickness in glaucoma patients with single-hemifield visual field defect .This was different from the results of Rao et al. (16) who suggested that structural changes occurred earlier than the reduction in retinal vessel density. But 
this difference could be attributed to the difference in disease etiology. Also, Richter et al. ${ }^{\left({ }^{(9)}\right)}$ considered that OCTA parameters had stronger associations with functional rather than structural measures of glaucoma.

Compared to traditional glaucoma staging system judged by VF, the results of PpVD could be a new glaucoma staging system judged by OCTA. In our study, we attempted to classify the stage of glaucoma based on the average of PpVD: stage 0 (no glaucoma) was determined when PpVD was 55 or above values (early glaucoma) with PpVD between 50-54\%. (Moderate glaucoma) with PpVD between 49-40 \% and (severe glaucoma) with PpVD is less 40. Of course, this new classification was based on the average value of $\mathrm{PpVD}$ in the whole area. We still need much more data to support this staging standard or more rational approach to categorize the stage of glaucoma.

The changes of PpVD had strongly positive correlation with MD, GCC thickness and RNFL thickness, while had negative correlation with PSD and $\mathrm{C} / \mathrm{D}$ ratio. We suggested that the changes of $\mathrm{PpVD}$ can add to both detecting and monitoring the progression of glaucoma.

\section{CONCLUSION}

It could be concluded that OCT-A is a promising technology that enabled us with qualitative data about the microvascular dropout in POAG, and it might further improve our understanding about the role of ocular blood flow changes in glaucoma. Longitudinal studies are needed to determine the complex relationship between progressive changes in retinal vasculature in cases of glaucoma, and both the structural and functional measurements.

\section{REFERENCES}

1. Harasymowycz P, Birt C, Gooi P et al. (2016): Medical Management of Glaucoma in the 21st Century from a Canadian Perspective. J Ophthalmol., 2016:6509809.

2. Tham Y, Li X, Wong T et al. (2014): Global prevalence of glaucoma and projections of glaucoma burden through 2040: a systematic review and meta- analysis. Ophthalmology, 121:2081-90.

3. Garway-Heath D, Lascaratos G, Bunce C et al. (2013): The United Kingdom Glaucoma Treatment Study: a multicenter, randomized, placebo-con-trolled clinical trial: design and methodology. Ophthalmology, 120:68-76.

4. Kerrigan-Baumrind L, Quigley $\mathrm{H}$, Pease $\mathrm{M}$ et al. (2000): Number of ganglion cells in glaucoma eyes compared with threshold visual field tests in the same persons. Invest Ophthalmol Vis Sci., 41:741-8.

5. Flammer J, Orgül S, Costa V et al. (2002): The impact of ocular blood flow in glaucoma. Prog Retin Eye Res., 21:359-393.

6. Hwang J, Konduru $\mathrm{R}$, Zhang $\mathrm{X}$ et al. (2012): Relationship among visual field, blood flow, and neural structure measurements in glaucoma. Invest Ophthalmol Vis Sci., 53:3020-3026.

7. Dastiridou A, Chopra V (2018): Potential applications of optical coherence tomography angiography in glaucoma. Curr Opin Ophthalmol., 29(3):226-233.

8. Yarmohammadi A, Zangwill L, Diniz-Filho A et al. (2016): Relationship between Optical Coherence Tomography Angiography Vessel Density and Severity of Visual Field Loss in Glaucoma. Ophthalmology, 123(12):2498-2508.

9. Hood D, Raza A, de Moraes C et al. (2013): Glaucomatous damage of the macula. Prog Retin Eye Res., 32: 11-21.

10. Leung C, Chan W, Yung W et al. (2015): Comparison of macular and peripapillary measurements for the detection of glaucoma: an optical coherence tomography study. Ophthalmology, 112:391-400.

11. Pollet-Villard F, Chiquet C, Romanet J et al. (2014): Structure-function relationships with spectral-domain optical coherence tomography retinal nerve fiber layer and optic nerve head measurements. Invest Ophthalmol Vis Sci., 55:2953-2962.

12. Lee J, Morales E, Sharifipour F et al. (2017): The relationship between central visual field sensitivity and macular ganglion cell/inner plexiform layer thickness in glaucoma. Br J Ophthalmol., 101:1052-1058.

13. Li Z, Xu Z, Liu Q et al. (2020): Comparisons of retinal vessel density and glaucomatous parameters in optical coherence tomography angiography. PLoS ONE, 15(6):234816.

14. Wang $X$, Jiang $C$, Ko $T$ et al. (2015): Correlation between optic disc perfusion and glaucomatous severity in patients with open angle glaucoma: an optical coherence tomography angiography study. Graefes Arch Clin Exp Ophthalmol., 253:1557-1564.

15. Yarmohammadi A, Zangwill L, Diniz-Filho A et al. (2016): Optical Coherence Tomography Angiography Vessel Density in Healthy, Glaucoma Suspect, and Glaucoma Eyes. Invest Ophthalmol Vis Sci., 57(9):451459.

16. Rao H, Pradhan $Z$, Weinreb $R$ et al. (2017): Vessel density and structural measurements of optical coherence tomography in primary angle closure and primary angle closure glaucoma. Am J Ophthalmology, 77:106-115.

17. Almbsut M, Abdelhameed A, Sabry D et al. (2020): Evaluation of Macular Vessels Density Changes in Patients with Primary Open Angle Glaucoma by Sweptsource Optical Coherence Tomography Angiography. ARC Journal of Ophthalmology, 5(1): 6-13.

18. Yarmohammadi A, Zangwill L, Diniz-Filho A et al. (2017): Peripapillary and Macular Vessel Density in Patients with Glaucoma and Single-Hemifield Visual Field Defect. Ophthalmology, 124(5):709-719.

19. Richter G, Madi I, Chu Z et al. (2018): Structural and Functional Associations of Macular Microcirculation in the Ganglion Cell- Inner Plexiform Layer in Glaucoma Using Optical Coherence Tomography Angiography. J Glaucoma, 27:281-290. 